\title{
Review Article: \\ Role of MicroRNAs in BCG Therapy by the Induction of Neutrophil Extracellular Traps in Bladder Cancer
}

\author{
Mohammad Mehdi Adibzadeh Sereshgi ${ }^{1}$ Q , Ali Mohammad Mohseni Majd ${ }^{1}$, Sahar Salimi ${ }^{1}$, Hassan Noorbazargan²*
}

1. Immunoregulation Research Center, Shahed University, Tehran, Iran.

2. Department of Biotechnology, School of Advanced Technologies in Medicine, Shahid Beheshti University of Medical Sciences, Tehran, Iran.

$\begin{gathered}\text { Use your device to scan } \\ \text { and read the article online }\end{gathered}$
py by the Induction of Neutrophil Extracellular Traps in Bladder Cancer. Immunoregulation. 2020; 3(1):17-28. http://dx.doi.
org/10.32598/Immunoregulation.3.1.3
d col http://dx.doi.org/10.32598/Immunoregulation.3.1.3

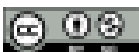

Article info:

Received: 17 Feb 2020

Accepted: 05 Apr 2020

Available Online: 01 Jul 2020

Keywords:

Bladder cancer, Bacillus Calmette-Guerin (BCG), Neutrophil extracellular traps (NETs), MicroRNAs (miRNAs)

\begin{abstract}
A B S T R A C T
The treatment of bladder cancer is usually performed by Bacillus Calmette-Guerin (BCG) instillation. BCG therapy is a common therapeutic method with fewer side effects compared with chemotherapy, radiotherapy, etc. BCG can also inhibit the progression and recurrence of bladder cancer by inducing apoptosis pathways, arrest cell cycle, autophagy, and neutrophil extracellular traps (NETs) formation. However, BCG therapy cannot be effective in the situation that the metastasis occurs. NETs are induced by BCG and help suppress the growth of tumor cells, especially in the primary stages of bladder cancer. Activated neutrophils can stimulate cellular pathways, such as autophagy and NETs release in the presence of microbial pathogenesis, inflammatory agents, and tumor cells. Autophagy can also regulate NETs formation and induce Reactive Oxygen Species (ROS) and NETs production. Moreover, miRNAs are key regulators of gene expression. These small non-coding RNAs are also considered as an essential factor in controlling tumor development. The interaction between BCG and miRNAs is still unclear. However, in the present study and for the first time, we intend to discuss the role of miRNAs in BCG therapy and how NETs formation can be effective on BCG performance to treat the bladder cancer.
\end{abstract}

\section{Introduction}

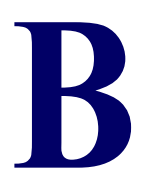

ladder Cancer (BC) arises from the tissues of the urinary bladder and is the $13^{\text {th }}$ common cause of mortality and the 9th most common cancer worldwide [1]. Conventional factors such as tumor grade, stage, and lymphatic and vascular extension, are prognostic indicators for BC. However, the currentlyused prognostic markers have a limited ability to predict progression, recurrence, metastasis, and response to therapy $[2,3]$. After the initial treatment of BC, a long-term follow-up is essential to prevent its recurrence. Generally, constant surveillance includes cystoscopy every 3 months for 2 years, then every 6 months for 2 years, and eventually annually, supposing no recurrence $[4,5]$. $\mathrm{BCG}$, as a live-attenuated strain of Mycobacterium bovis, is similar to M. tuberculosis in antigenic composition and has been utilized for treating bladder cancer [6]. In this respect, immune responses have a crucial role to

\footnotetext{
* Corresponding Author:
}

Hassan Noorbazargan, PhD.

Address: Department of Biotechnology, School of Advanced Technologies in Medicine, Shahid Beheshti University of Medical Sciences, Tehran, Iran. Phone: +98 (911) 3335669

E-mail: h.noorbazargan@gmail.com 
combat tumors. Neutrophils are the first leukocytes that fight tumors and can produce some special compositions that are called Neutrophil Extracellular Traps (NETs) [7]. Also, autophagy is a cellular destruction procedure in which damaged organelles and cytosolic components are degraded into autophagolysosome, which is created by the fusion of phagosome and lysosome [8]. Additionally, several studies showed that BCG (Bacillus CalmetteGuerin) therapy can induce NETs and autophagy which prevent tumor growth or metastasis $[9,10]$.

Another effective factor is epigenetic agents. microRNAs (miRNAs) are the noncoding small RNAs that are identified to regulate expression of genes involved in the control of proliferation, development, and apoptosis [11]. Moreover, various studies indicate that miRNAs can contribute to suppressing the growth of tumor cells. Thus, the imbalance of miRNAs gene expression could result in excessive proliferation. In this regard, miRNAs play a crucial role in prognosis of the bladder cancer, especially the initial phase [12]. Furthermore, miRNAs can stimulate autophagy and neutrophil extracellular traps (NETs) formation in neutrophils and inhibit tumor metastasis [13].

BCG therapy could prevent recurrence and progression of tumors in bladder cancer. However, dysregulation of some cellular and molecular processes such as autophagy pathway and NETs formation could result in metastasis of $\mathrm{BC}$ [14]. The growth and proliferation of tumor cells are facilitated by dysregulation of miRNAs gene expression and autophagy process; also the NETs formation could act in favor of tumor invasion [15]. Interestingly, therapeutic options may serve as regulator agents to inhibit cancer progression and improve BCG therapy efficacy through a complex network of miRNAs, autophagy, and NETs [16-18]. Although BCG has been administrated for the treatment of BC since many years ago, its therapeutic effect should be more evaluated to elucidate this matter. Thus, the present review study is to discuss the effect of miRNAs on BCG therapy via NETs formation in bladder cancer.

\section{BCG therapy in bladder cancer}

For the first time, the efficacy of BCG therapy for bladder cancer has been shown by Morales et al. [19]. Intravesical BCG is established now as a criterion of care for the high-risk, non-muscle invasive disease, including high-grade papillary tumors (stage Ta), carcinoma in situ (stage Tis, also called CIS), and lamina-propria-invasive tumors (stage T1) [20, 21].
In these conditions, treatment with BCG could be associated with a lower risk of recurrence com $\neg$ pared with transurethral resection alone. Also, the risk of progression to invasive disease would be reduced by using BCG therapeutic approach [22-24]. Besides, BCG compares favorably with intravesical chemotherapy with respect to decreasing the risk of recurrence; however, the toxicity of BCG can be more intense [25-27].

\section{Neutrophil role in physiological situations and cancer}

Neutrophils are the key part of the innate immune system and have an undeniable role in the control of infectious diseases. These predominant leukocytes are among the first blood cells seen in an inflammatory site [28]. NETs are a network of chromatin structures with the related enzymes, including elastase, myeloperoxidase, and cathepsin $\mathrm{G}$, which were released by stimulation of Phorbol Myristate Acetate (PMA), carcinogenesis substance. NETs can trap, neutralize, and kill the extracellular bacteria, viruses, fungi, and parasites. Moreover, NET exclusion occurs initially via a cell death process termed NETosis [29]. This process begins with the interruption of the nuclear envelope and continues with chromatin decondensation into the cytoplasm of intact cells. Also, NETiosis can occur after the secretion of nuclear chromatin that is accompanied by the release of granule proteins via degranulation [30].

\section{NETosis definition}

The role of NETs in tumor progression remains poorly understood. The obtained evidence from some studies suggests a potential relationship between tumor progression and intra-tumoral NET deposition in both experimental models and patients with cancer [31-33]. Zychlinsky et al. evaluated the presence of tumor-associated neutrophils (TANs) and NETs in surgical resection specimens of 8 patients with sarcoma as determined by positive staining for extracellular myeloperoxidase (MPO) and found that $25 \%$ of these patients ( 2 patients), exhibited intra-tumor NET deposition [31]. These two patients developed early relapse after performing post-neoadjuvant chemotherapy and surgery, although the place was not defined in the study [31]. Therefore, it seems that Ewing sarcoma cells can stimulate TANs to release NETs. The capability of tumor cells to induce neutrophils to generate NETs has been displayed in several tumor types. This phenomenon indicates the possibility that NETs play a fundamental role in the biology of tumors [34]. 
In this regard, Demers et al. demonstrated that several tumor types, including lung, mammary, and hematologic neoplasms can stimulate circulating neutrophils to produce NETs [34]. The evidence presented in the literature suggests that NETs may promote tumor progression within the primary tumor [35].

As previously stated, NETs have usually powerful adhesive characteristics, which empower them to bind pathogens and platelets. Thereby, NETs may also provide intravascular networks assisting tumor cell adhesion in angiogenesis and metastasis. Neutrophils can suppress circulating tumor cells, especially under inflammatory circumstances, which remarks the role of NETs in this procedure [36-38]. Additional direct evidence arises from a current in vitro study demonstrating that pulmonary carcinoma cells display about five-fold adhesion increase to NETs compared with non-stimulated neutrophil monolayers $[32,39]$.

Another main aspect is the role of neutrophils in the cancer microenvironment. As regards this matter, some studies recently performed in the field of neutrophil roles in tumor microenvironment suggest that neutrophils exhibit crucial plasticity which could be turned to N1 (antitumoral) or $\mathrm{N} 2$ (protumoral) phenotype in response to the tumor context $[40,41]$. Tumor-associated N2 neutrophils are identified by high expression of VEGF (vascular endothelial growth factor), CXCR4 (CXC chemokine receptor 4), gelatinase $\mathrm{B}$, and MMP9 (matrix metalloproteinase-9) and can be induced on exposure to high TGF- $\beta$ (transforming growth factor-beta) levels. On the other hand, N1 neutrophils express immunopotentiating cytokines and chemokines such as IFN- $\gamma$ (interferon-gamma), CXCR3, and low levels of arginase. They are also induced on TGF- $\beta$ blockade and can eliminate cancer cells $[40,42]$.

As regards, Berger-Achituv et al. study showed that NETs have either pro- or anti-tumor function, depending on factors such as tumor microenvironment and type of cancer. For instance, within the microenvironment of the tumor, TGF- $\beta$ can induce TANs with pro-tumorigenic features. However, TANs produce pro-inflammatory cytokines and have tumoricidal activity without TGF- $\beta$ [40]. In addition, neutrophils can enhance tumor growth through the production of matrix metalloproteinase (MMP)-9 that inhibits tumor cell apoptosis in the respiratory tract and increases tumor angiogenesis and neovascularization [43, 44]. Nevertheless, neutrophils can also have a cytotoxic effect on tumor cells by generating many types of reactive oxygen species (ROS) [45, 46]. Notably, a study demonstrated that neutrophils inhibit metastatic seeding by secreting hydrogen peroxide in a mouse model of breast cancer [47]. Neutrophils also secret defensins, which have anti-angiogenetic characteristics and can lyse cancer cells, recruit dendritic cells (DCs) as an antigen-presenting cell [48].

NETs are thought to have anti-tumorigenic effects, for example through activating immune responses and killing tumor cells. On the other hand, NETs could have a pro-tumorigenic function by promoting metastases. NETs may physically take tumor cells and inhibit their dissemination to adjacent tissues. Various components of NETs are cytotoxic to tumor cells. MPO was demonstrated to destroy B-16 melanoma cells and prevent their growth in mice after implantation [49]. Interestingly, patients with MPO deficiency probably have a high incidence of cancer (7/14 patients, 50\%) [50]. NETs can eradicate activated endothelial cells based on a conducted study from Gupta and their colleagues, may by histones, damaging tumor-feeding blood vessels [51]. NE produced by TANs cleaves Cyclin E to other isoforms with lower molecular weight and therefore facilitates their presentation to CTLs [52]. NETs have a modulatory role and establish a bridge between innate and adaptive immunity by activating plasmacytoid dendritic cells through TLR9, an intracellular receptor that preferentially binds DNA. NETs can prime T cells by TCR signaling that implicates direct contact [53].

Alternatively, NETs that contain different proteases could represent a pro-tumorigenic activity through degradation of the extracellular matrix and increase metastasis. NETs may also create a hurdle between cancer cells and the immune system, thereby collaborating with cancer cells to evade immune recognition. Consequently, it has been reported that patients with the metastatic disease showed NETs formation relapse that may refer to the pro-tumorigenic mechanism of NETs [31]. Moreover, recent evidence indicates that neutrophils from certain donors can eliminate cancer cells in a cell-specific manner and the neutrophil killing of cancer cells probably is improved by the treatment of $\beta$-glucan, making neutrophil a persuadable candidate for cancer immunotherapy [54]. Various studies that trigger neutrophilia through prolonged G-CSF (granulocyte-colony stimulating factor) treatment in tumors usually shows a change from a chronic mode to an acute inflammatory situation and an antitumor effect [55]. Notably, mammary tumor cell lines stimulate NETosis in vitro, but there is no strong evidence for the NET formation in vivo [56]. On the other hand, some studies have demonstrated that NETiosis can fight cancer metastasis [42] 
One underlying mechanism for metastasis suppression seems to be the NET-mediated capture of migrating tumor cells, particularly at sites of inflammation, which can be blocked with NE and protein-arginine deiminase type 4 (PAD4) inhibitors [57]. Therefore, targeting NETs through these pathways could be a promising therapeutic option for cancers. In the next section, we will discuss the role of BCG therapy in bladder cancer and the interaction between NETs and BCG for the treatment of bladder cancer [57].

In sum, neutrophil-induced NETs act as an inhibitor for the development of tumor metastasis through elastase, MPO, and other enzymes. In contrast, little evidence demonstrates that produced NETs from the TANs in the microenvironment of tumors can result in the progress of tumor cells.

\section{BCG therapy and the role of neutrophils}

As mentioned above, BCG administration into the bladder provides a localized infection that involves both attachment and then internalization into normal and malignancy urothelial cells through the fibronectin process mediated by integrin adhesion molecules [58-60]. Recent studies have demonstrated that neutrophils can migrate to the bladder after BCG stimulates bladder epithelial cells to secrete chemokines. Also, another study shows that neutrophils have an important role in the anticancer outcome of BCG therapy. With regard to this issue, Suttman et al. reported that neutrophils could be the reason for the positive outcome of BCG therapy in a mouse bladder tumor model [61].

They found that BCG therapy has no effect after depletion of neutrophils that results in a reduction in survival compared with non-depleted controls. Neutrophils release interleukin (IL)-8, macrophage inflammatory-1 $\alpha$ (MIP-1 $\alpha$ ), and growth-regulated oncogene- $\alpha$ (GRO- $\alpha$ ) when they are stimulated with BCG in vitro. Therefore, BCG-induced chemokine secretion by neutrophils recruits macrophages, which eventually recruits $\mathrm{T}$ cells. According to these findings, Suttman et al. suggest that BCG administration can result in the influx of neutrophils that coordinate the subsequent macrophages and $\mathrm{T}$ cell recruitment via the release of chemokines [61]. Interestingly, in consideration of Suttman et al. results, it is proposed that the BCG-induced antitumor responses are mediated by activated T cells, whereas neutrophils recruit other immune cells with indirect mechanism [60, 61]. Additionally, neutrophils have a direct antitumor immunity through the production of soluble TRAIL ( tumor necrosis factor-related apoptosis-inducing ligand) in the bladder environment [60].
In another study, Liu et al. evaluated the formation of NETs by the induction of BCG instillation. They reported that tumor cell proliferation was inhibited by treatment with NETs as well as cytotoxicity of NETs on tumor cells. Their results demonstrated that BCG-induced NETs promote dose- and time-dependent apoptosis of tumor cells and G0/G1 phase arrest. Liu et al. study findings demonstrated that BCG-activated tumors stimulate more NETs compared with non-activated ones. Also, neutrophil adhesion and NETs release are increased by stimulation with supernatant of activated cells which, represent a significant role for cytokines. Their results also suggest that bladder cancer cells induce NETs via TNF- $\alpha$ and IL-8 secretion following BCG stimulation. Eventually, they concluded that BCG-induced NETs suppress tumors through multiple mechanisms, including cytotoxic effects, induction of apoptosis, and cell cycle arrest.

Some studies have shown that NETs could suppress migration and invasion of tumor cells by the induction of apoptosis and exertion of cytotoxicity mechanism on tumor cells [9]. Besides, the role of neutrophils has been proven for $\mathrm{T}$ cells trafficking to the bladder after BCG perfusion [61]. According to IFN- $\gamma$ cytokine production or activation of CD8+ T and natural killer (NK) cells, CD4+ $\mathrm{T}$ cells are the main contributors in BCG therapy $[61,62]$. Furthermore, NETs could prime T cells and activate DCs. NETs treatment upregulate CD4 expression in vitro, and CD3+ and CD14+ cells in tumors that could be an important index for potentiating immunity. Generally, the presence of monocyte, T helper (Th)1 cells, and CTLs (cytotoxic $\mathrm{T}$ lymphocyte) in the environment of tumor could result in tumor regression, and also a favorable prognosis [9]. It has been demonstrated that NETs have either tumor pro- or anti-tumor activity. Therefore, some agents such as cytokine profiles in the microenvironment and cancer type are the determinative subject for progression or suppression of tumor cells $[63,64]$. At high concentrations, the release of MPO and NE as important components of NETs have a cytotoxic effect on tumor cells, but reducing the release may result in the conversion of anti-tumor to pro-tumor function $[65,66]$. Thus, NETs have different roles based on variations in multiple stimuli, neutrophil action sites, and induction with BCG or others [9].

Altogether, neutrophils are induced by BCG activation to form NETs. In other words, the direct role of BCG-induced NETs is indicated by the cytotoxic effect, apoptosis induction, cell cycle arrest, and inhibition of tumor cells migration into the bladder environment. Besides, NETs also have an indirect role through stimulation immunity and recruitment of $\mathrm{T}$ cells and macrophages to prevent tumor growth. 
mTOR (mammalian target of rapamycin) as a serine/ threonine-protein kinase can form two different protein complexes known as mTORC1 and mTORC2. Also, AMP-dependent protein kinase (AMPK) is the key sensor of cellular energy status, which activates the autophagy pathway [15-20]. Class I and class III PI3K (phosphatidylinositol 3-kinase) pathways are the substantial regulators of autophagy. Class I PI3K activates mTORC1 and inhibits the start of autophagy. On the contrary, class III PI3K can directly induce autophagy [67]. The autophagy pathway has a key role in tumor suppression and progression at the early and late stages of bladder cancer [68].

According to various studies, mTORC1 has a fundamental role in the regulation of several cellular mechanisms, such as autophagy and differentiation of neutrophilic precursor cells. These mechanisms could be ceased by using pharmacological inhibition of mTORC1-induced autophagy or p38 Mitogen-Activated Protein Kinase (MAPK) [69].

It has been demonstrated that neutrophils are primed by autophagy for increased NETs formation, which is notable for appropriate neutrophil effector functions during sepsis. Neutrophils have a potency to increase autophagy induction in patients who survived sepsis. On the other hand, there is an abnormal autophagy function in neutrophils isolated from patients who could not survive sepsis. However, in murine models of sepsis, the autophagy reinforcement improved survival through a NET-dependent mechanism [70].

Notably, autophagy and ROS production as two main regulators of NETosis have a close correlation with each other. Autophagy induction can occur through ROS burst, which in turn is necessary to maintain effective ROS production [71].

Remijsen et al. studied the role of autophagy and ROS production in forming NETosis. Their results demonstrated that a combination of ROS production and autophagy is required for PMA-induced-NET formation in human neutrophils. Suppression of either NADPH (nicotinamide adenine dinucleotide phosphate) oxidase or autophagy could prevent the chromatin decondensation that is crucial for NETosis, resulting in apoptotic cell death. Additionally, there is no NADPH oxidase activity in neutrophils isolated from patients with chronic granulomatous disease (CGD). These neutrophils cannot produce NETs [72] (Figure 1).

Also, another study showed that neutrophils from patients with AGH (acute gouty arthritis) display autopha- gy-mediated spontaneous NET release [73]. Currently, it has also been demonstrated that reduced expression levels of Atg5 (autophagy-related gene 5) as a component of the autophagy system interplays with the low capacity of neutrophils to form NETs when TLR2 ligand stimulation occurs in aged mice. This issue suggests that it may represent a major role of autophagy in maintaining the mechanism of NETs [74].

On the other hand, contradictory data have also been published concerning the contribution of autophagy in NET release. Particularly, Atg5-knockout mouse neutrophils that display decreased autophagic activity kept the capacity to release extracellular DNA. Moreover, PI3K can prevent NET formation inhibition by human neutrophils [75]. Consequently, it may exist an autophagyindependent NETosis pathway $[75,76]$.

Altogether, activated neutrophil may promote autophagic activity and NET formation. Also, autophagy induces NET formation. But in relation to cancers, especially bladder cancer, the ability of autophagy to induce NETs formation has yet to be determined and no research in the literature discussed it. However, according to similar studies, it seems that autophagy-induced NETs formation would happen in the tumor microenvironment through tumor-associated neutrophils (N2). Still, further investigations should be performed to clarify the matter. In the next section, the role of autophagy in cancer and angiogenesis will be discussed

\section{miRNAs role in BCG therapy}

miRNAs are a branch of noncoding small RNAs that can have different effects on oncogenesis by acting as oncogene or tumor suppressor in a microenvironmentdependent manner [77, 78]. Various studies performed on the role of miRNAs in bladder cancer demonstrated that some miRNAs are overexpressed and the gene expression of other miRNAs are down-regulated. Gottardo et al. evaluated the expression of miRNAs in 27 bladder specimens. They found that the expression of some miRNAs such as miR17-5p, miR23a, miR23b, miR26b, miR103-1, miR185, miR203, miR205, miR221, and miR223 remarkably increased in bladder cancer. In other words, the function of a specific miRNA depends on its target genes. Thus, the upregulated miRNAs effect on oncogenes can be considered as tumor-suppressing miRNAs, and downregulated miRNAs effect on tumor suppressor genes can be considered as onco-miRNAs [79].

Another study showed that the expression of $4 \mathrm{miR}$ NAs (miR199a-3p, miR195, miR133a, and miR30a-3p) 
is downregulated in tumors; however, these miRNAs usually act as tumor suppressors [78]. On the contrary, miR200c and miR21 are upregulated in bladder cancer tissues and could be an agent for the progression of bladder cancer $[80,81]$. The expression of miRNAs is detected using molecular techniques such as microarray or deep sequencing in patients with bladder cancer. Many types of samples are obtained from clinical tissue specimens, fluids, body, and bladder cancer cell lines [82-84].

BCG therapy has been demonstrated with the ability to prevent the occurrence and progression of cancer through various mechanisms, such as autophagy inhibition. Some studies have indicated that miR9-3, miR1242, miR124-3, and miR137 were frequently methylated in the initiation phase of cancers which can utilize as potential biomarkers for bladder cancer diagnosis [85]. Interestingly, no research and review articles have been published about the effect of BCG on miRNAs profile expression by searching "Bacillus Calmette-Guerin", "miRNA", terms or "BCG", "miRNA", terms in the $\mathrm{MeSH}$ in this respect. However, several studies about the role of BCG and miRNAs in infectious diseases have been conducted. For example, one study evaluated the alteration of immune-related miR142-3p in macrophage RAW264.7 cells in treatment with BCG infection. Their results showed that miR142-3p can negatively regulate the production of pro-inflammatory mediators of IL-6, TNF- $\alpha$, and NF- $\kappa$ B (NF- $\kappa \mathrm{B} 1)$ in the macrophages by post-transcriptionally down-regulating IRAK-1 protein expression [86]. Also, another study demonstrated that M. bovis BCG induces toll-like receptor 2 (TLR2)-dependent miR155 expression, which establishes signaling cross-talk among MAPKs, $\mathrm{PKC} \delta$ (protein kinase $\mathrm{C} \delta$ ), and PI3K also the recruitment of c-ETS and NF- $\mathrm{KB}$ as miR155 promoter. Finally, they indicated that the cellular reprogramming was organized by miR155 during immune responses to mycobacterial infection [87].

In sum, BCG can stimulate immune responses and triggers signaling molecular pathways by making interaction with miRNAs. It seems that BCG instillation can affect miRNAs expression in bladder cancer tissue. Nevertheless, more research must be conducted to clarify this interaction in bladder cancer.

So far, little research has done on how miRNAs can affect the NETosis process. However, some studies have conducted on the interaction between miRNA expression and neutrophil. As regards this issue, one study showed that neutrophil-associated miR99b-5p, miR191$5 p$, and miR197-3p transcript levels were remarkably lower in Mycobacterium tuberculosis (MT) infections.
Differential expression of miRNAs in neutrophils can predominantly affect the signaling pathways leading to cytokine productions. The reduced expression in MT cases could indicate a lack of inhibition on signaling pathways, which may result in elevated production of pro-inflammatory cytokines such as IFN- $\gamma$ [88].

As was discussed previously, IFN- $\gamma$ is a crucial cytokine in immune responses to microbial infections. In this regard, a study demonstrated the significance of IFN- $\gamma$ role in NETs function when lung neutrophils of mice are infected by Streptococcus pneumonia, Staphylococcus aureus, and Escherichia coli. Their results revealed that decreased formation of NETs in IFN- $\gamma-$ deficient mice could increase in S. pneumonia bacterial number $[89,90]$.

According to Gantier et al. study, we prepared a list, including 48 miRNAs that are expressed in neutrophils. We found that miRNAs have a key role in the regulation of neutrophil biology. Of the expressed miRNAs in neutrophil, miR1 and miR133 are down-regulated in patients with myeloproliferative disorders. miR223 has also been demonstrated as the main miRNAs expressed by human granulocytes (CD15). One study reported an essential role of miR223 in neutrophil differentiation by evaluating miR223-deficient mice [91]. Ward et al. indicated that miRNAs of miR34b, miR328, miR483-3p, miR491-3p, miR595, and miR1281 are up-regulated when neutrophils are treated with granulocyte-macrophage-colony-stimulating factor (GM-CSF).

In other words, GM-CSF treatment delays apoptosis and senescence (Table 1) [92]. IL-8 as a CXC chemokine ligand has a crucial function in the recruitment of human leukocytes, especially neutrophils, and is also produced by various immune cells such as neutrophils, macrophages, and epithelial cells stimulated with TNF- $\alpha$. miR17 can directly target IL- 8 mRNA; therefore, miR17 inhibition could drastically increase IL-8 production [93]. On the other hand, miR155 can increase IL-8 secretion from neutrophils of patients with cystic fibrosis by the suppression of SHIP expression. Elevated miR155 levels can straightly decrease levels of SHIP-1, which ordinarily destabilize IL-8 mRNA via Akt signaling $[78,94]$. Hence, therapeutic approaches should focus on decreasing miR155 and increasing miR17 levels, which could dampen IL-8 production by neutrophils.

Some studies have also determined that neutrophils can polarize and migrate towards the center of tumor cells that highly express these chemotactic factors [95, 96]. As soon as the chemokine reaches the maximum pro- 
Table 1. miRNAs expression in neutrophils

\begin{tabular}{cl}
\hline miRNAs & Expression \\
\hline miR1 & Downregulation \\
\hline miR133 & Downregulation \\
miR34b & Upregulation \\
miR328 & Upregulation \\
miR483-3p & Upregulation \\
miR491-3p & Upregulation \\
miR595 & Upregulation \\
\hline miR1281 & Upregulation \\
\hline
\end{tabular}

ImiunoRegulation

duction zone, the gradient of chemotactic concentration vanishes. The chemotactic stimulus can establish NETs formation when many receptors get occupied [73]. A study by using intravital microscopy in tumors could show that neutrophils move to the tumor and form NETs (personal communication). In the tumor microenvironment, these structures are often related to the processes that favor metastasis $[32,97,98]$. A study in mice also indicated that NETs facilitate the metastatic capacity of tumor cells, in other words, they favor their migration [99]. However, the extent to which NETs can affect the function of other immune cells in the tumor microenvironment has not been directly demonstrated [100].

There are relatively scarce data about the role of miRNAs in neutrophil biology in the literature. Different expressions of miRNAs in the tumor microenvironment

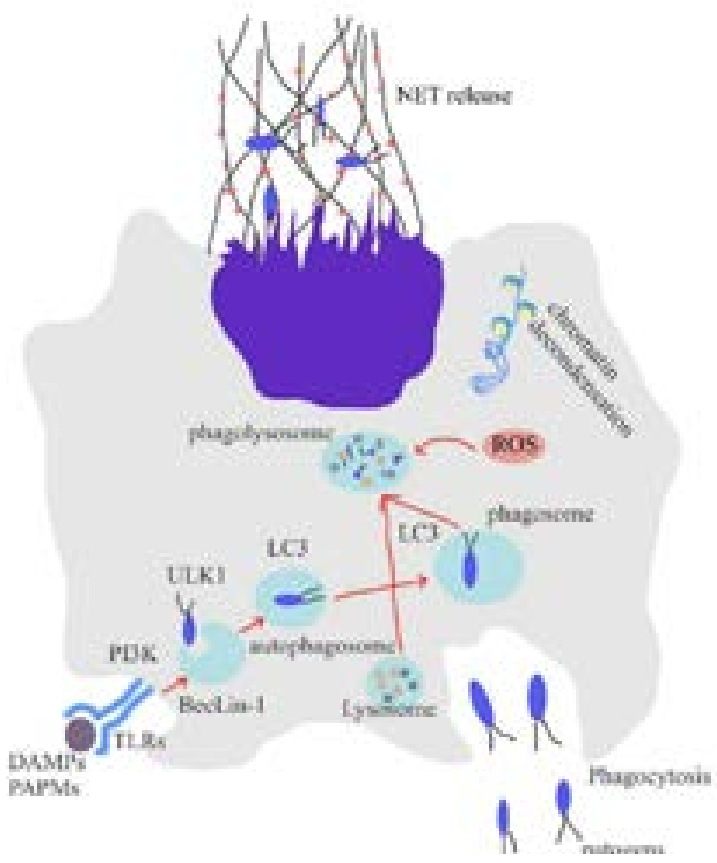

Figure 1. Role of autophagy and reactive oxygen species (ROS) production in forming NETosis tin decondensation that is crucial for NETosis, resulting in apoptotic cell death. NETosis can eliminate infections and tumors 
could be a useful option for the prognosis, diagnosis, and treatment of various cancers, especially bladder cancer.

\section{Therapeutic options}

Today, molecular targeting as a novel therapeutic approach is considered for improving survival and prognosis in patients with BC $[101,102]$. Various studies have shown that abnormally-expressed miRNAs are involved in $\mathrm{BC}$ progression by acting as oncogenes or tumor suppressors. Recent reports have demonstrated that the noninvasive detection of miRNAs in body fluids, including urine and blood of $\mathrm{BC}$ patients, can be used to improve $\mathrm{BC}$ prognosis and diagnosis [103, 104]. Thereby, the recognition of dysregulated miRNAs to promote clinical applications in BC is necessary. In this respect, one study identified various dysregulated miRNAs in BC [105]. Some miRNAs play major roles in autophagy regulation. For instance, the findings of some studies revealed that miR99a-5p exhibits a tumor suppressor role via targeting mTOR in BC [106]. Thereby, the performed studies have indicated a promising role of the miRNAs in BC therapy [101]. On the other hand, NETs formation in tumor regulation by miRNAs can increase the NETs formation in patients with $\mathrm{BC}[9,107,108]$.

Generally, NETs formation can facilitate BCG performance in BC treatment. NETs can suppress tumor activity through specific mechanisms, including the mTOR signaling pathway and production of ROS by neutrophils $[9,60]$. Thus, promoting NETs performance by miRNAs can be utilized to improve BCG therapy in patients with $\mathrm{BC}$. In addition to the diagnostic usefulness of miRNAs, engineering and designing miRNAs could also be a suitable therapeutic strategy to eliminate tumor cells of BC through targeting mTOR or other mediators, which have a key role in this field. Additional efforts are essential to assess the therapeutic roles of candidate miRNAs and their interaction between NETs formation. Eventually, further investigations are necessary to further clarify novel RNA networks in bladder cancer cells.

BCG therapy is usually prescribed for patients with non-muscle invasive bladder cancer. The results of this review study revealed that miRNAs have an impressive role to induce NETs formation which can also promote BCG therapy in patients with BC. Also, BCG-induced NETs can perform the cytotoxic effect, apoptosis induction, cell cycle arrest, and inhibition of tumor cells migration into the bladder environment. Moreover, neutrophils can prime $\mathrm{T}$ cells and activate DCs to robust immune responses against tumor growth. Therefore, the utilization of miRNAs network as a therapeutic approach may reinforce BCG function through induction of NETs formation by stimulating neutrophils.

\section{Ethical Considerations}

Compliance with ethical guidelines

All ethical principles were considered in this article.

Funding

This research did not receive any specific grant from funding agencies in the public, commercial, or not profit sectors.

\section{Authors' contributions}

All authors contributed in preparing this article.

\section{Conflicts of interest}

The authors declared no conflict of interest.

\section{References}

[1] von der Maase H, Sengelov L, Roberts JT, Ricci S, Dogliotti L, Oliver $\mathrm{T}$, et al. Long-term survival results of a randomized trial comparing gemcitabine plus cisplatin, with methotrexate, vinblastine, doxorubicin, plus cisplatin in patients with bladder cancer. Journal of Clinical Oncology. 2005; 23(21):4602-8. [DOI:10.1200/JCO.2005.07.757] [PMID]

[2] Bellmunt J, von der Maase H, Mead GM, Skoneczna I, De Santis M, Daugaard G, et al. Randomized phase III study comparing paclitaxel/cisplatin/gemcitabine and gemcitabine/cisplatin in patients with locally advanced or metastatic urothelial cancer without prior systemic therapy: EORTC intergroup study 30987. Journal of Clinical Oncology. 2012; 30(10):1107-13. [DOI:10.1200/JCO.2011.38.6979] [PMID] [PMCID]

[3] Wang W, Wang H, Teng X, Wang H, Mao C, Teng R, et al. Clonal analysis of bilateral, recurrent, and metastatic papillary thyroid carcinomas. Human Pathology. 2010; 41(9):1299-309. [DOI:10.1016/j.humpath.2010.02.008] [PMID]

[4] Barton Grossman H, Natale RB, Tangen CM, Speights VO, Vogelzang NJ, Trump DL, et al. Neoadjuvant chemotherapy plus cystectomy compared with cystectomy alone for locally advanced bladder cancer. The New England Journal of Medicine. 2003; 349(9):859-66. [DOI:10.1056/NEJMoa022148] [PMID]

[5] Necchi A, Pond GR, Giannatempo P, Di Lorenzo G, Eigl BJ, Locke J, et al. Cisplatin-based first-line therapy for advanced urothelial carcinoma after previous perioperative cisplatinbased therapy. Clinical Genitourinary Cancer. 2015; 13(2):17884. [DOI:10.1016/j.clgc.2014.08.010] [PMID] [PMCID]

[6] Kaufmann E, Sanz J, Dunn JL, Khan N, Mendonça LE, Pacis A, et al. BCG educates hematopoietic stem cells to generate pro- 
tective innate immunity against tuberculosis. Cell. 2018; 172(12):176-90.E19. [DOI:10.1016/j.cell.2017.12.031] [PMID]

[7] Hisada Y, Grover SP, Maqsood A, Houston R, Ay C, Noubouossie DF, et al. Neutrophils and neutrophil extracellular traps enhance venous thrombosis in mice bearing human pancreatic tumors. Haematologica. 2020; 105(1):218-25. [DOI:10.3324/ haematol.2019.217083] [PMID] [PMCID]

[8] Mizushima N. Autophagy: Process and function. Genes \& Development. 2007; 21(22):2861-73. [DOI:10.1101/ gad.1599207] [PMID]

[9] Liu K, Sun E, Lei M, Li L, Gao J, Nian X, et al. BCG-induced formation of neutrophil extracellular traps play an important role in bladder cancer treatment. Clinical Immunology. 2019; 201:414. [DOI:10.1016/j.clim.2019.02.005] [PMID]

[10] Vita F, Siracusano S, Abbate R, Ciciliato S, Borelli V, Soranzo $\mathrm{MR}$, et al. BCG prophylaxis in bladder cancer produces activation of recruited neutrophils. The Canadian Journal of Urology. 2011; 18(1):5517-23. [PMID]

[11] Baehrecke EH. miRNAs: Micro managers of programmed cel death. Current Biology. 2003; 13(12):R473-R5. [DOI:10.1016/ S0960-9822(03)00405-6]

[12] Usuba W, Urabe F, Yamamoto Y, Matsuzaki J, Sasaki H, Ichikawa $\mathrm{M}$, et al. Circulating miRNA panels for specific and early detection in bladder cancer. Cancer Science. 2019; 110(1):408-19. [DOI:10.1111/cas.13856] [PMID] [PMCID]

[13] Fu LL, Wen X, Bao JK, Liu B. MicroRNA-modulated autophagic signaling networks in cancer. The International Journal of Biochemistry \& Cell Biology. 2012; 44(5):733-6. [DOI:10.1016/j. biocel.2012.02.004] [PMID]

[14] Eskelinen EL. The dual role of autophagy in cancer. Current Opinion in Pharmacology. 2011; 11(4):294-300. [DOI:10.1016/j. coph.2011.03.009] [PMID]

[15] Chen PS, Su JL, Hung MC. Dysregulation of microRNAs in cancer. Journal of Biomedical Science. 2012; 19(1):90. [DOI:10.1186/1423-0127-19-90] [PMID] [PMCID]

[16] Buffen K, Oosting M, Quintin J, Ng A, Kleinnijenhuis J, Kumar V, et al. Autophagy controls BCG-induced trained immunity and the response to intravesical BCG therapy for bladder cancer. PLoS Pathogens. 2014; 10(10):e1004485. [DOI:10.1371/ journal.ppat.1004485] [PMID] [PMCID]

[17] Heneghan HM, Miller N, Kerin MJ. MiRNAs as biomarkers and therapeutic targets in cancer. Current Opinion in Pharmacology. 2010; 10(5):543-50. [DOI:10.1016/j. coph.2010.05.010] [PMID]

[18] Kiselyov A, Bunimovich-Mendrazitsky S, Startsev V. Treatment of non-muscle invasive bladder cancer with Bacillus Calmette-Guerin (BCG): Biological markers and simulation studies. BBA Clinical. 2015; 4:27-34. [DOI:10.1016/j.bbacli.2015.06.002] [PMID] [PMCID]

[19] Morales A, Eidinger D, Bruce AW. Intracavitary Bacillus Calmette-Guerin in the treatment of superficial bladder tumors. The Journal of Urology. 1976; 116(2):180-2. [DOI:10.1016/ S0022-5347(17)58737-6]

[20] Babjuk M, Oosterlinck W, Sylvester R, Kaasinen E, Böhle A, Palou-Redorta J, et al. EAU guidelines on non-muscle-invasive urothelial carcinoma of the bladder, the 2011 update.
European Urology. 2011; 59(6):997-1008. [DOI:10.1016/j. eururo.2011.03.017] [PMID]

[21] Craig Hall M, Chang SS, Dalbagni G, Pruthi RS, Seigne JD, Skinner EC, et al. Guideline for the management of nonmuscle invasive bladder cancer (stages Ta, T1, and Tis): 2007 update. The Journal of Urology. 2007; 178(6):2314-30. [DOI:10.1016/j. juro.2007.09.003] [PMID]

[22] Han RF, Pan JG. Can intravesical bacillus Calmette-Guérin reduce recurrence in patients with superficial bladder cancer? A meta-analysis of randomized trials. Urology. 2006; 67(6):121623. [DOI:10.1016/j.urology.2005.12.014] [PMID]

[23] Shelley MD, Kynaston H, Court J, Wilt TJ, Coles B, Burgon K, et al. A systematic review of intravesical bacillus Calmette-Guérin plus transurethral resection vs transurethral resection alone in Ta and T1 bladder cancer. BJU International. 2001; 88(3):209-16. [DOI:10.1046/j.1464-410x.2001.02306.x] [PMID]

[24] Sylvester R, van der Meijden A, Lamm D. Intravesical Bacillus Calmette-Guerin reduces the risk of progression in patients with superficial bladder cancer: A metaanalysis of the published results of randomized clinical trials. The Journal of Urology. 2002; 168(5):1964-70. [DOI:10.1097/00005392-200211000-00016]

[25] Redelman-Sidi G, Glickman MS, Bochner BH. The mechanism of action of BCG therapy for bladder cancer-a current perspective. Nature Reviews Urology. 2014; 11(3):153-62. [DOI:10.1038/nrurol.2014.15] [PMID]

[26] Shang PF, Kwong J, Wang ZP, Tian J, Jiang L, Yang KH, et al. Intravesical Bacillus Calmette-Guérin versus epirubicin for $\mathrm{Ta}$ and T1 bladder cancer. Cochrane Database of Systematic Reviews. 2011; (5):Art.No:CD006885. [DOI:10.1002/14651858. CD006885.pub2] [PMID]

[27] Shelley MD, Wilt TJ, Court J, Coles B, Kynaston H, Mason MD. Intravesical bacillus Calmette-Guérin is superior to mitomycin $\mathrm{C}$ in reducing tumour recurrence in high-risk superficial bladder cancer: A meta-analysis of randomized trials. BJU International. 2004; 93(4):485-90. [DOI:10.1111/j.1464410X.2003.04655.x] [PMID]

[28] Mantovani A, Cassatella MA, Costantini C, Jaillon S. Neutrophils in the activation and regulation of innate and adaptive immunity. Nature Reviews Immunology. 2011; 11(8):519-31. [DOI:10.1038/nri3024] [PMID]

[29] Brinkmann V, Zychlinsky A. Neutrophil extracellular traps: Is immunity the second function of chromatin? Journal of Cell Biology. 2012; 198(5):773-83. [DOI:10.1083/jcb.201203170] [PMID] [PMCID]

[30] Branzk N, Papayannopoulos V. Molecular mechanisms regulating NETosis in infection and disease. Seminars in Immunopathology. 2013; 35(4):513-30. [DOI:10.1007/s00281-013-03846] [PMID] [PMCID]

[31] Berger-Achituv S, Brinkmann V, Abu Abed U, Kühn LI, Ben-Ezra J, Elhasid R, et al. A proposed role for neutrophil extracellular traps in cancer immunoediting. Frontiers in Immunology. 2013; 4:48. [DOI:10.3389/fimmu.2013.00048] [PMID] [PMCID]

[32] Cools-Lartigue J, Spicer J, McDonald B, Gowing S, Chow S, Giannias B, et al. Neutrophil extracellular traps sequester circulating tumor cells and promote metastasis. The Journal of Clinical Investigation. 2013; 123(8):3446-58. [DOI:10.1172/JCI67484] [PMID] [PMCID] 
[33] Sangaletti S, Tripodo C, Vitali C, Portararo P, Guarnotta C, Casalini $\mathrm{P}$, et al. Defective stromal remodeling and neutrophil extracellular traps in lymphoid tissues favor the transition from autoimmunity to lymphoma. Cancer Discovery. 2014; 4(1):11029. [DOI:10.1158/2159-8290.CD-13-0276] [PMID]

[34] Demers M, Krause DS, Schatzberg D, Martinod K, Voorhees JR, Fuchs TA, et al. Cancers predispose neutrophils to release extracellular DNA traps that contribute to cancer-associated thrombosis. Proceedings of the National Academy of Sciences of the United States of America. 2012; 109(32):13076-81. [DOI:10.1073/pnas.1200419109] [PMID] [PMCID]

[35] Cools-Lartigue J, Spicer J, Najmeh S, Ferri L. Neutrophil extracellular traps in cancer progression. Cellular and Molecular Life Sciences. 2014; 71(21):4179-94. [DOI:10.1007/s00018-014-16833] [PMID] [PMCID]

[36] Liang Sh, Hoskins M, Khanna P, Kunz RF, Dong C. Effects of the tumor-leukocyte microenvironment on melanoma-neutrophil adhesion to the endothelium in a shear flow. Cellular and Molecular Bioengineering. 2008; 1(2-3):189-200. [DOI:10.1007/ s12195-008-0016-8] [PMID] [PMCID]

[37] McDonald B, Spicer J, Giannais B, Fallavollita L, Brodt P, Ferri LE. Systemic inflammation increases cancer cell adhesion to hepatic sinusoids by neutrophil mediated mechanisms. International Journal of Cancer. 2009; 125(6):1298-305. [DOI:10.1002/ ijc.24409] [PMID]

[38] Spicer JD, McDonald B, Cools-Lartigue JJ, Chow SC, Giannias $B$, Kubes $\mathrm{P}$, et al. Neutrophils promote liver metastasis via mac1-Mediated interactions with circulating tumor cells. Cancer Research. 2012; 72(16):3919-27. [DOI:10.1158/0008-5472.CAN11-2393] [PMID]

[39] Erpenbeck L, Schön MP. Neutrophil extracellular traps: Protagonists of cancer progression? Oncogene. 2017; 36(18):248390. [DOI:10.1038/onc.2016.406] [PMID]

[40] Fridlender ZG, Sun J, Kim S, Kapoor V, Cheng G, Ling L, et al. Polarization of tumor-associated neutrophil phenotype by TGF- $\beta$ : "N1" versus “N2" TAN. Cancer Cell. 2009; 16(3):183-94. [DOI:10.1016/j.ccr.2009.06.017] [PMID] [PMCID]

[41] Piccard H, Muschel RJ, Opdenakker G. On the dual roles and polarized phenotypes of neutrophils in tumor development and progression. Critical Reviews in Oncology/Hematology. 2012; 82(3):296-309. [DOI:10.1016/j.critrevonc.2011.06.004] [PMID]

[42] Powell DR, Huttenlocher A. Neutrophils in the tumor microenvironment. Trends in Immunology. 2016; 37(1):41-52. [DOI:10.1016/j.it.2015.11.008] [PMID] [PMCID]

[43] Acuff HB, Carter KJ, Fingleton B, Lee Gorden D, Matrisian LM. Matrix metalloproteinase- 9 from bone marrow-derived cells contributes to survival but not growth of tumor cells in the lung microenvironment. Cancer Research. 2006; 66(1):25966. [DOI:10.1158/0008-5472.CAN-05-2502] [PMID] [PMCID]

[44] Masson V, De La Ballina LR, Munaut C, Wielockx B, Jost M, Maillard C, et al. Contribution of host MMP-2 and MMP-9 to promote tumor vascularization and invasion of malignant keratinocytes. The FASEB Journal. 2005; 19(2):1-17. [DOI:10.1096/ fj.04-2140fje] [PMID] [PMCID]

[45] Dallegri F, Ottonello L, Ballestrero A, Dapino P, Ferrando F, Patrone F, et al. Tumor cell lysis by activated human neutrophils: Analysis of neutrophil-delivered oxidative attack and role of leukocyte function-associated antigen 1. Inflammation. 1991; 15(1):15-30. [DOI:10.1007/BF00917906] [PMID]

[46] Lichtenstein A. Stimulation of the respiratory burst of murine peritoneal inflammatory neutrophils by conjugation with tumor cells. Cancer Research. 1987; 47(9):2211-7. [PMID]

[47] Granot Z, Henke E, Comen EA, King TA, Norton L, Benezra $R$. Tumor entrained neutrophils inhibit seeding in the premetastatic lung. Cancer Cell. 2011; 20(3):300-14. [DOI:10.1016/j. ccr.2011.08.012] [PMID] [PMCID]

[48] Al-Benna S, Shai Y, Jacobsen F, Steinstraesser L. Oncolytic activities of host defense peptides. International Journal of Molecular Sciences. 2011; 12(11):8027-51. [DOI:10.3390/ ijms12118027] [PMID] [PMCID]

[49] Odajima T, Onishi M, Hayama E, Motoji N, Momose Y, Shigematsu A. Cytolysis of B-16 melanoma tumor cells mediated by the myeloperoxidase and lactoperoxidase systems. Biological Chemistry. 1996; 377(11):689-93. [DOI:10.1515/ bchm3.1996.377.11.689]

[50] Lanza F, Giuliani AL, Amelotti F, Spisani S, Traniello S, Castoldi G. Depressed neutrophil-mediated tumor cell cytotoxicity in subjects affected by hereditary myeloperoxidase deficiency and secondary neoplasia. Haematologica. 1988; 73(5):355-8. [PMID]

[51] Saffarzadeh M, Juenemann C, Queisser MA, Lochnit G, Barreto G, Galuska SP, et al. Neutrophil extracellular traps directly induce epithelial and endothelial cell death: A predominant role of histones. PloS One. 2012; 7(2):e32366. [DOI:10.1371/ journal.pone.0032366] [PMID] [PMCID]

[52] Mittendorf EA, Alatrash G, Qiao N, Wu Y, Sukhumalchandra $\mathrm{P}$, St. John LS, et al. Breast cancer cell uptake of the inflammatory mediator neutrophil elastase triggers an anticancer adaptive immune response. Cancer Research. 2012; 72(13):3153-62. [DOI:10.1158/0008-5472.CAN-11-4135] [PMID] [PMCID]

[53] Tillack K, Breiden P, Martin R, Sospedra M. T lymphocyte priming by neutrophil extracellular traps links innate and adaptive immune responses. The Journal of Immunology. 2012; 188(7):3150-9. [DOI:10.4049/jimmunol.1103414] [PMID]

[54] Yan J, Kloecker G, Fleming C, Bousamra M, Hansen R, Hu $X$, et al. Human polymorphonuclear neutrophils specifically recognize and kill cancerous cells. OncoImmunology. 2014; 3(7):e950163. [DOI:10.4161/15384101.2014.950163] [PMID] [PMCID]

[55] Souto JC, Vila L, Brú A. Polymorphonuclear neutrophils and cancer: Intense and sustained neutrophilia as a treatment against solid tumors. Medicinal Research Reviews. 2011; 31(3):311-63. [DOI:10.1002/med.20185] [PMID]

[56] Demers M, Wagner DD. NETosis: A new factor in tumor progression and cancer-associated thrombosis. Seminars in Thrombosis and Hemostasis. 2014; 40(3):277-83. [DOI:10.1055/s-0034-1370765] [PMID] [PMCID]

[57] Cedervall J, Olsson AK. NETosis in cancer. Oncoscience. 2015; 2(11):900-1. [DOI:10.18632/oncoscience.264] [PMID] [PMCID]

[58] Becich MJ, Carroll Sh, Ratliff TL. Internalization of Bacille Calmette-Guerin by bladder tumor cells. The Journal of Urology. 1991; 145(6):1316-24. [DOI:10.1016/S0022-5347(17)38622-6]

[59] Luo Y, Szilvasi A, Chen X, DeWolf WC, O'Donnell MA. A novel method for monitoring Mycobacterium bovis BCG 
trafficking with recombinant BCG expressing green fluorescent protein. Clinical and Diagnostic Laboratory Immunology. 1996; 3(6):761-8. [DOI:10.1128/CDLI.3.6.761768.1996] [PMID] [PMCID]

[60] Simons MP, O'Donnell MA, Griffith TS. Role of neutrophils in BCG immunotherapy for bladder cancer. Urologic Oncology: Seminars and Original Investigations. 2008; 26(4):341-5. [DOI:10.1016/j.urolonc.2007.11.031] [PMID] [PMCID]

[61] Suttmann H, Riemensberger J, Bentien G, Schmaltz D, Stöckle $M$, Jocham D, et al. Neutrophil granulocytes are required for effective Bacillus Calmette- Guérin immunotherapy of bladder cancer and orchestrate local immune responses. Cancer Research. 2006; 66(16):8250-7. [DOI:10.1158/0008-5472.CAN06-1416] [PMID]

[62] Böhle A, Brandau S. Immune mechanisms in Bacillus Calmette-Guerin immunotherapy for superficial bladder cancer. The Journal of Urology. 2003; 170(3):964-9. [DOI:10.1097/01. ju.0000073852.24341.4a] [PMID]

[63] Brincks EL, Risk MC, Griffith TS. PMN and anti-tumor immunity--the case of bladder cancer immunotherapy. Seminars in Cancer Biology. 2013; 23(3):183-9. [DOI:10.1016/j.semcancer.2013.02.002] [PMID]

[64] Zhou SL, Zhou ZJ, Hu ZQ, Huang XW, Wang Z, Chen EB, et al. Tumor-associated neutrophils recruit macrophages and T-regulatory cells to promote progression of hepatocellular carcinoma and resistance to Sorafenib. Gastroenterology. 2016; 150(7):1646-58.E17. [DOI:10.1053/j.gastro.2016.02.040] [PMID]

[65] Di Carlo E, Forni G, Lollini PL, Colombo MP, Modesti A, Musiani $\mathrm{P}$. The intriguing role of polymorphonuclear neutrophils in antitumor reactions. Blood. 2001; 97(2):339-45. [DOI:10.1182/ blood.V97.2.339] [PMID]

[66] Houghton AM. The paradox of tumor-associated neutrophils Fueling tumor growth with cytotoxic substances. Cell Cycle. 2010; 9(9):1732-7. [DOI:10.4161/cc.9.9.11297] [PMID]

[67] Sridharan S, Jain K, Basu A. Regulation of autophagy by kinases. Cancers. 2011; 3(2):2630-54. [DOI:10.3390/cancers3022630] [PMID] [PMCID]

[68] Singh SS, Vats S, Chia AYQ, Tan TZ, Deng Sh, Ong MS, et al. Dual role of autophagy in hallmarks of cancer. Oncogene. 2018; 37(9):1142-58. [DOI:10.1038/s41388-017-0046-6] [PMID]

[69] Simon HU, Friis R, Tait SWG, Ryan KM. Retrograde signaling from autophagy modulates stress responses. Science Signaling. 2017; 10(468):eaag2791. [DOI:10.1126/ scisignal.aag2791] [PMID]

[70] Park SY, Shrestha S, Youn YJ, Kim JK, Kim SY, Kim HJ, et al. Autophagy primes neutrophils for neutrophil extracellular trap formation during sepsis. American Journal of Respiratory and Critical Care Medicine. 2017; 196(5):577-89. [DOI:10.1164/ rccm.201603-0596OC] [PMID]

[71] Filomeni G, De Zio D, Cecconi F. Oxidative stress and autophagy: The clash between damage and metabolic needs. Cell Death \& Differentiation. 2015; 22(3):377-88. [DOI:10.1038/ cdd.2014.150] [PMID] [PMCID]

[72] Remijsen Q, Kuijpers TW, Wirawan E, Lippens S, Vandenabeele P, Vanden Berghe T. Dying for a cause: NETosis, mechanisms behind an antimicrobial cell death modality. Cell Death \& Differentiation. 2011; 18(4):581-8. [DOI:10.1038/cdd.2011.1] [PMID] [PMCID]

[73] Skendros P, Mitroulis I, Ritis K. Autophagy in neutrophils: From granulopoiesis to neutrophil extracellular traps. Frontiers in Cell and Developmental Biology. 2018; 6:109. [DOI:10.3389/ fcell.2018.00109] [PMID] [PMCID]

[74] Xu F, Zhang C, Zou Z, Fan EKY, Chen L, Li Y, et al. Agingrelated Atg5 defect impairs neutrophil extracellular traps formation. Immunology. 2017; 151(4):417-32. [DOI:10.1111/ imm.12740] [PMID] [PMCID]

[75] Germic N, Stojkov D, Oberson K, Yousefi Sh, Simon HU. Neither eosinophils nor neutrophils require ATG 5-dependent autophagy for extracellular DNA trap formation. Immunology. 2017; 152(3):517-25. [DOI:10.1111/ imm.12790] [PMID] [PMCID]

[76] Pieterse E, Rother N, Yanginlar C, Hilbrands LB, van der Vlag J. Neutrophils discriminate between lipopolysaccharides of different bacterial sources and selectively release neutrophil extracellular traps. Frontiers in Immunology. 2016; 7:484 [DOI:10.3389/fimmu.2016.00484] [PMID] [PMCID]

[77] Lin JF, Chen PC, Hwang TI. Autophagy modulation by dysregulated micrornas in human bladder cancer. Urological Science. 2019; 30(2):46-52. http://www.e-urol-sci.com/text. asp?2019/30/2/46/255165

[78] Adibzadeh Sereshgi MM, Abdollahpour-Alitappeh M, Mahdavi M, Ranjbar R, Ahmadi K, Taheri RA, et al. Immunologic balance of regulatory $\mathrm{T}$ cell/ $\mathrm{T}$ helper 17 responses in gastrointestinal infectious diseases: Role of miRNAs. Microbial Pathogenesis. 2019; 131:135-43. [DOI:10.1016/j.micpath.2019.03.029] [PMID]

[79] Gottardo F, Liu CG, Ferracin M, Calin GA, Fassan M, Bassi $\mathrm{P}$, et al., editors. Micro-RNA profiling in kidney and bladder cancers. Urologic Oncology: Seminars and Original Investigations. 2007; 25(5):387-92. [DOI:10.1016/j.urolonc.2007.01.019] [PMID]

[80] Cheng Y, Zhang X, Li P, Yang C, Tang J, Deng X, et al. MiR200c promotes bladder cancer cell migration and invasion by directly targeting RECK. Onco Targets and Therapy. 2016 9:5091-9. [DOI:10.2147/OTT.S101067] [PMID] [PMCID]

[81] Zhang HH, Qi F, Cao YH, Zu XB, Chen MF. Expression and clinical significance of microRNA-21, maspin and vascular endothelial growth factor-C in bladder cancer. Oncology Letters. 2015; 10(4):2610-6. [DOI:10.3892/ ol.2015.3540] [PMID] [PMCID]

[82] Han Y, Chen J, Zhao X, Liang C, Wang Y, Sun L, et al. MicroRNA expression signatures of bladder cancer revealed by deep sequencing. PloS One. 2011; 6(3):e18286. [DOI:10.1371/journal.pone.0018286] [PMID] [PMCID]

[83] Ichimi T, Enokida H, Okuno Y, Kunimoto R, Chiyomaru $\mathrm{T}$, Kawamoto K, et al. Identification of novel microRNA targets based on microRNA signatures in bladder cancer. International Journal of Cancer. 2009; 125(2):345-52. [DOI:10.1002/ijc.24390] [PMID]

[84] Tölle A, Jung M, Rabenhorst S, Kilic E, Jung K, Weikert S. Identification of microRNAs in blood and urine as tumour markers for the detection of urinary bladder cancer. Oncology Reports. 2013; 30(4):1949-56. [DOI:10.3892/or.2013.2621] [PMID] 
[85] Shimizu T, Suzuki H, Nojima M, Kitamura H, Yamamoto E, Maruyama R, et al. Methylation of a panel of microRNA genes is a novel biomarker for detection of bladder cancer. European Urology. 2013; 63(6):1091-100. [DOI:10.1016/j. eururo.2012.11.030] [PMID]

[86] Xu G, Zhang Z, Wei J, Zhang Y, Zhang Y, Guo L, et al. microR-142-3p down-regulates IRAK-1 in response to Mycobacterium bovis BCG infection in macrophages. Tuberculosis. 2013; 93(6):606-11. [DOI:10.1016/j.tube.2013.08.006] [PMID]

[87] Ghorpade DS, Leyland R, Kurowska-Stolarska M, Patil SA, Balaji KN. MicroRNA-155 is required for Mycobacterium bovis BCG-mediated apoptosis of macrophages. Molecular and Cellular Biology. 2012; 32(12):2239-53. [DOI:10.1128/MCB.0659711] [PMID] [PMCID]

[88] van Rensburg IC, du Toit L, Walzl G, du Plessis N, Loxton AG. Decreased neutrophil-associated miRNA and increased B-cell associated miRNA expression during tuberculosis. Gene. 2018; 655:35-41. [DOI:10.1016/j.gene.2018.02.052] [PMID]

[89] Gantier MP. The not-so-neutral role of microRNAs in neutrophil biology. Journal of Leukocyte Biology. 2013; 94(4):575-83. [DOI:10.1189/jlb.1012539] [PMID]

[90] Yamada M, Gomez JC, Chugh PE, Lowell CA, Dinauer MC, Dittmer DP, et al. Interferon- $\gamma$ production by neutrophils during bacterial pneumonia in mice. American Journal of Respiratory and Critical Care Medicine. 2011; 183(10):1391-401. [DOI:10.1164/rccm.201004-0592OC] [PMID] [PMCID]

[91] Liu Q, Li A, Tian Y, Wu JD, Liu Y, Li T, et al. The CXCL8CXCR1/2 pathways in cancer. Cytokine \& Growth Factor Reviews. 2016; 31:61-71. [DOI:10.1016/j.cytogfr.2016.08.002] [PMID] [PMCID]

[92] Jones SA, Wolf M, Qin S, Mackay CR, Baggiolini M. Different functions for the interleukin 8 receptors (IL-8R) of human neutrophil leukocytes: NADPH oxidase and phospholipase D are activated through IL-8R1 but not IL-8R2. Proceedings of the National Academy of Sciences of the United States of America. 1996; 93(13):6682-6. [DOI:10.1073/pnas.93.13.6682] [PMID] [PMCID]

[93] Karin N. Chemokines and cancer: New immune checkpoints for cancer therapy. Current Opinion in Immunology. 2018; 51:140-5. [DOI:10.1016/j.coi.2018.03.004] [PMID]

[94] Yousefi Sh, Stojkov D, Germic N, Simon D, Wang X, Benarafa C, et al. Untangling "NETosis" from NETs. European Journal of Immunology. 2019; 49(2):221-7. [DOI:10.1002/eji.201747053] [PMID]

[95] Azevedo PO, Paiva AE, Santos GSP, Lousado L, Andreotti JP, Sena IFG, et al. Cross-talk between lung cancer and bones results in neutrophils that promote tumor progression. Cancer Metastasis Reviews. 2018; 37(4):779-90. [DOI:10.1007/s10555018-9759-4] [PMID] [PMCID]

[96] Weiß E, Kretschmer D. Formyl-peptide receptors in infection, inflammation, and cancer. Trends in Immunology. 2018; 39(10):815-29. [DOI:10.1016/j.it.2018.08.005] [PMID]

[97] Park J, Wysocki RW, Amoozgar Z, Maiorino L, Fein MR, Jorns J, et al. Cancer cells induce metastasis-supporting neutrophil extracellular DNA traps. Science Translational Medicine. 2016; 8(361):361ra138. [DOI:10.1126/scitranslmed.aag1711] [PMID] [PMCID]
[98] Pasquier J, Vidal F, Hoarau-Véchot J, Bonneau C, Daraï E, Touboul C, et al. Surgical peritoneal stress creates a pro-metastatic niche promoting resistance to apoptosis via IL-8. Journal of Translational Medicine. 2018; 16(1):271. [DOI:10.1186/s12967018-1643-z] [PMID] [PMCID]

[99] Tohme S, Yazdani HO, Al-Khafaji AB, Chidi AP, Loughran $\mathrm{P}$, Mowen $\mathrm{K}$, et al. Neutrophil extracellular traps promote the development and progression of liver metastases after surgical stress. Cancer Research. 2016; 76(6):1367-80. [DOI:10.1158/0008-5472.CAN-15-1591] [PMID] [PMCID]

[100] Gonzalez-Aparicio M, Alfaro C. Influence of interleukin-8 and Neutrophil Extracellular Trap (NET) formation in the tumor microenvironment: Is there a pathogenic role? Journal of Immunology Research. 2019; 2019:6252138. [DOI:10.1155/2019/6252138] [PMID] [PMCID]

[101] Blanca A, Cheng L, Montironi R, Moch H, Massari F, Fiorentino M, et al. Mirna expression in bladder cancer and their potential role in clinical practice. Current Drug Metabolism. 2017; 18(8):712-22. [DOI:10.2174/1389200218 666170518164507] [PMID]

[102] Zheng Y, Tan F, Wang L, Xu N, Mou H. Primary primitive neuroectodermal tumor of the urinary bladder: A case report and literature review. Medical Oncology. 2011; 28 Suppl 1:S388S91. [DOI:10.1007/s12032-010-9680-3] [PMID]

[103] Jin N, Jin X, Gu X, Na W, Zhang M, Zhao R. Screening biomarkers of bladder cancer using combined miRNA and mRNA microarray analysis. Molecular Medicine Reports. 2015; 12(2):3170-6. [DOI:10.3892/mmr.2015.3739] [PMID]

[104] Zhang Y, Zhang D, Lv J, Wang Sh, Zhang Q. MiR-125a-5p suppresses bladder cancer progression through targeting FUT4. Biomedicine \& Pharmacotherapy. 2018; 108:1039-47. [DOI:10.1016/j.biopha.2018.09.100] [PMID]

[105] Chen L, Yuan L, Wang G, Cao R, Peng J, Shu B, et al. Identification and bioinformatics analysis of miRNAs associated with human muscle invasive bladder cancer. Molecular Medicine Reports. 2017; 16(6):8709-20. [DOI:10.3892/mmr.2017.7726] [PMID] [PMCID]

[106] Lin JF, Tsai TF, Lin YC, Chen HE, Chou KY, Hwang TI. Benzyl isothiocyanate suppresses IGF1R, FGFR3 and mTOR expression by upregulation of miR-99a-5p in human bladder cancer cells. International Journal of Oncology. 2019; 54(6):210616. [DOI:10.3892/ijo.2019.4763] [PMID]

[107] Boone BA, Orlichenko L, Schapiro NE, Loughran P, Gianfrate GC, Ellis JT, et al. The receptor for advanced glycation end products (RAGE) enhances autophagy and neutrophil extracellular traps in pancreatic cancer. Cancer Gene Therapy. 2015; 22(6):326-34. [DOI:10.1038/cgt.2015.21] [PMID] [PMCID]

[108] Jagannath C, Lindsey DR, Dhandayuthapani S, Xu Y, Hunter Jr RL, Tony Eissa N. Autophagy enhances the efficacy of BCG vaccine by increasing peptide presentation in mouse dendritic cells. Nature Medicine. 2009; 15(3):267-76. [DOI:10.1038/ nm.1928] [PMID] 